\title{
Images de l'excès : Shakespeare et l'art graphique des pays du Nord
}

Josée Nuyts-Giornal

\section{(2) OpenEdition}

\section{Journals}

\section{Édition électronique}

URL : http://journals.openedition.org/shakespeare/1065

DOI : 10.4000/shakespeare.1065

ISSN : 2271-6424

\section{Éditeur}

Société Française Shakespeare

\section{Édition imprimée}

Date de publication : 1 novembre 2007

Pagination : 133-153

ISBN : 2-9521475-4-X

\section{Référence électronique}

Josée Nuyts-Giornal, «Images de l'excès : Shakespeare et l'art graphique des pays du Nord », Actes des congrès de la Société française Shakespeare [En ligne], 25 | 2007, mis en ligne le 10 février 2008, consulté le 20 avril 2019. URL : http://journals.openedition.org/shakespeare/1065 ; DOI : 10.4000/ shakespeare.1065 


\section{Shakespeare et l'excès}

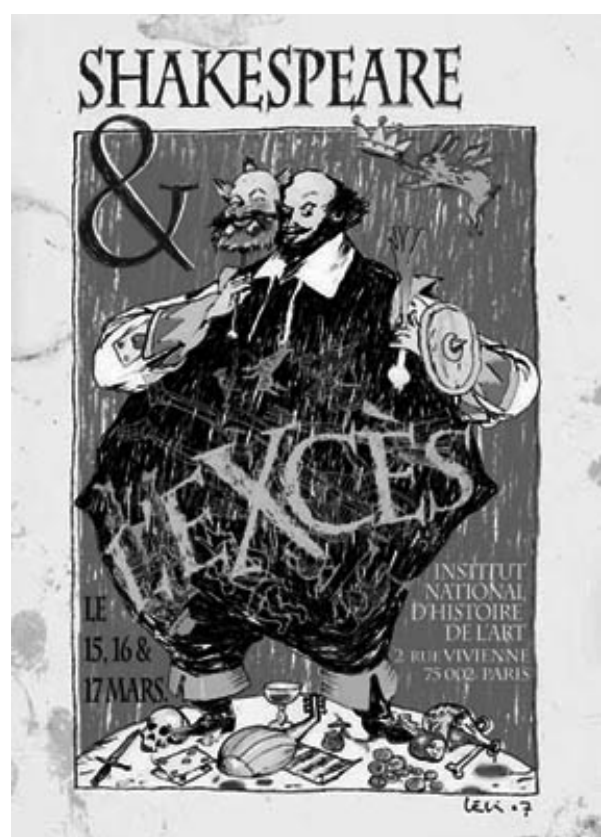

actes du Congrès

organisé par la

SOCIÉTÉ FRANÇAISE SHAKESPEARE

les 15,16 et 17 mars 2007

textes réunis par

Pierre KAPITANIAK

sous la direction de

Jean-Michel DÉPRATS 
COUVERTURE :

Edouard Lekston, Falstaff, 2007

conception graphique et logo

Pierre Kapitaniak

\section{(C) 2007 Société Française Shakespeare \\ Institut du Monde Anglophone}

Université de Paris III - Sorbonne Nouvelle

5 rue de l'École de Médecine 75006 Paris

www.societefrancaiseshakespeare.org

Tous droits de traduction, de reproduction et d'adaptation réservés pour tous les pays 


\title{
IMAGES DE L'EXCÈS : SHAKESPEARE ET L'ART GRAPHIQUE DES PAYS DU NORD
}

\author{
Josée NUYTS-GIORNAL
}

\begin{abstract}
L'art pictural du Nord s'attache aux images de l'excès et de la transgression qui sont une façon de renouer avec la catharsis antique. Le thème humaniste du miroir de la folie est le dénominateur commun de beaucoup de ces images qui se rattachent à la thématique du monde à l'envers et à l'art de l'allusion satirique et à la parodie burlesque. Dans As You Like It, Shakespeare semble nous inviter à imaginer une interface entre la satire morale de la gravure et le texte dramatique, lorsqu'Orlando accuse Monsieur Mélancolie (Jaques) d'emprunter aux lieux communs imagés des tissus peints pour réprimander les dérèglements de Dame Monde (III.ii.268-69). Nous étudierons les récurrences de certains des motifs du discours visuel sur l'excès au sein du texte dramatique et plus particulièrement dans Hamlet, Timon of Athens, Antony and Cleopatra et Coriolanus. Car, si le texte shakespearien adopte les bribes de ce discours, le dramaturge peut aussi en subvertir le sens et en sonder les limites.
\end{abstract}

Early modern pictorial art of the North pretended to mend morals with images of transgression and excess. The humanist theme of the mirror of folly was the leitmotiv of most of these images, which can be put into relation with the German and Dutch narrenliteratur, the topic of the world upside down and with social satire. In As You Like It, Shakespeare hints at a possible interaction between this visual medium of moral satire and the dramatic text when Orlando mocks Monsieur Melancholy's use of moral maxims found in painted-cloths in order to rail against the misconduct of mistress the world (III.ii.268-69). The playwright draws on this visual discourse on excess and its moral commonplaces in many of his plays. We will study the recurrence of some of these motifs in Hamlet, Timon of Athens, Antony and Cleopatra, and Coriolanus.

$\mathrm{D}$

ans l'art pictural des pays du Nord, la représentation de l'excès et de la transgression affirme un espoir de renouer avec la fonction éthique et cathartique du théâtre antique. Le thème humaniste du miroir de la folie est le dénominateur commun de beaucoup de ces images qui se rattachent à la tradition de la Narrenliteratur allemande et néerlandaise proche de l'univers de la fatrasie médiévale, à la thématique du monde à l'envers ou du contemptus mundi, à la tradition grotesque et obscène ainsi qu'à l'allusion satirique et à la parodie burlesque. Souvent exposée par un bouffon sage, sage en ce qu'il reconnait sa propre folie, et qui occupe la place excentrée et privilégiée de persona satirique, l'image de l'excès apparaît comme un exemple négatif dont il convient de prendre connaissance pour mieux éviter d'y sombrer ensuite. Le thème du miroir de la folie parcourt le Xvi ${ }^{e}$ et le début du XvII ${ }^{e}$ siècle. Ainsi, John Milton rend-il hommage à Edmund Spenser au motif que sa poésie visuelle surpasserait par sa valeur didactique l'enseignement de Jean Duns Scot ou de Thomas d'Aquin quand il dépeint les dangers de la luxure dans les jardins d'Acrasie de façon telle que le chevalier Guyon 
ne peut que s'abstenir en connaissance de cause (« [that he] might see and know, and yet abstain ${ }^{1}$ ). Lorsque, dans As You Like It, Orlando accuse Monsieur Mélancolie (Jaques) d'emprunter ses figures aux lieux communs mis en images sur les tissus peints dans le but de réprimander les dérèglements de Dame Monde (III.ii.268-69), on retrouve en partie la traditionnelle imagerie humaniste s'efforçant de moraliser le thème de l'excès. Mais, tout en adoptant et adaptant quelques bribes de ce discours visuel, il peut aussi en subvertir le sens et en sonder les limites. L'étude d'un corpus d'images liées au miroir de la folie et au thème des cinq sens, grâce auxquels on peut appréhender le monde mais qui sont aussi le biais par lequel succomber à ses tentations, pourra servir à éclairer certains emprunts shakespeariens, dans Hamlet et Timon of Athens en particulier. Il s'agira donc ici de mettre au jour des schémas de pensée communs entre le texte dramatique et un certain type de « discours » visuel.

\section{L’image peinte comme discours sur l'excès}

Shakespeare fait souvent allusion aux images peintes sur des tentures domestiques, images qu'il désigne sous le terme générique de « painted cloth ». Largement répandues à l'époque, elles nous renseignent sur les thématiques propres à ce support graphique particulier dont seuls quelques rares exemplaires ont été conservés. Le cliché de l'image parlante, la «speaking picture ${ }^{2}$ » qui renvoie à l'« ut pictura poesis » d'Horace se retrouve dans la réplique de Jaques à Orlando : «I answer you right painted cloth, from whence you have studied your questions » (As You Like It, III.ii.268-69) ${ }^{3}$. Cela implique un certain mépris face à un support qui associait maximes et lieux communs mis en images et Jaques félicite ironiquement Orlando pour sa perspicacité, dans la mesure où il a bien vu où le mélancolique puisait son inspiration: «You have a nimble wit; I think it was made of

\footnotetext{
${ }^{1}$ John Milton, Aeropagitica, éd. Tony Davies, in Selected Shorter Poems and Prose, Londres, Routledge, 1988, (1 ${ }^{\text {re }}$ éd. 1644), p. 100-107.

${ }^{2}$ Voir à ce propos Michael Bath, Speaking Pictures; English Emblem Books and Renaissance Culture, Londres Longman, 1994.

3 Voir aussi Richard II : "You would have thought / [...] / [...] that all the walls /With painted imagery had said at once... » (Richard II, v.ii.15-16), ou encore « painted rhetoric » (LLL, Iv.ii.237) William Shakespeare, The Complete Works, éd. Stanley Wells et Gary Taylor, Oxford, Clarendon Press, 1998.
} 
Atlanta's heels. Will you sit down with me, and we two will rail against our mistress the world, and all our misery ? » (III.ii.268-69). La leçon de morale donnée à une humanité prise au piège de ses passions et des tentations du monde semble bien associée à la technique un peu rudimentaire $\mathrm{du}$ «painted cloth». Dans The Rape of Lucrece, les proverbes et les adages restent de peu de poids face à la violence du désir de Tarquin : «My will is strong past reason's weak removing. / Who fears a sentence or an old man's saw / Shall by a painted cloth be kept in awe » (v. 243-45). L'idée d'exemplum édifiant se retrouve dans l'épilogue ironique de Pandare à la fin de Troilus and Cressida: " Good traders in the flesh set this in your painted cloths ; / [...] / Your eyes, half out, - weep out at Pandar's fall » (Épilogue [in-quarto de 1609], 14-16). Les déconvenues du personnage sont censées prévenir les personnes du métier des dangers inhérents à la profession d'entremetteur. En même temps, l'art dramatique et l'art pictural se superposent dans leurs desseins respectifs ${ }^{4}$. Ces commentaires ironiques, qui prennent à contre-pied l'orthodoxie du "painted cloth » avertissant le spectateur contre le danger des excès propres à la nature humaine, renvoient au sentiment de banalité qu'elle pouvait à juste titre inspirer à l'époque. Les murs de la Renaissance anglaise étaient en effet ornés de fresques représentant des héros et des personnages célèbres de l'Antiquité qui étaient mêlés à tout un bric-à-brac de sentences, de drôleries, d'histoires de fils prodigue ${ }^{5}$ et autres tableaux allégoriques figurant les vicissitudes humaines. Ainsi, au terme d'une énumération d'aphorismes moraux n'ayant pas su amener l'homme à plus de sagesse, Robert Burton, dans The Anatomy of Melancholy, invite le lecteur à poursuivre : "Look for more in Isocrates, Seneca, Plutarch, Epictetus, etc., and for defect, consult with cheese trenchers and painted-cloths ${ }^{6}$. »Et, pour ce qui est des sujets des tissus peints

\footnotetext{
${ }^{4}$ Dans Love's Labour's Lost, Costard ne s'y trompe pas lorsqu'il prévient un personnage qu'il ne tient pas son rôle, celui d'Alexandre le Grand en l'occurrence, l'un des Neuf Preux, et s'expose ainsi à être effacé du «painted cloth » - «You will be scraped out of the painted cloth for this » (v.ii.571-72). John Nevinson rapporte cette scène à un tissu peint d'Amersham et Antony Wells-Cole fait le lien avec une série de gravures par Philips de Galle également repris par Thomas Trevelyon. Voir A. Wells-Cole, Art and Decoration in Elizabethan and Jacobean England, Londres, Yale University Press, 1997, p. 200.

5 Voir The Merry Wives of Windsor, "'Tis painted about with the story of the Prodigal ». (IV.iv.6-8)

${ }^{6}$ Robert Burton, The Anatomy of Melancholy, éd. Holbrook Jackson, Everyman's Library, 3 vol., Londres, Dent, 1977 vol 2, Pt.2 Sec 3 Mem.7, p. 205. À propos de l'imagerie des « cheese trenchers », voir Malcolm Jones, « The Neglected Genres of Popular Verse 1480-
} 
anglais, il s'avère que les artistes se sont effectivement inspirés des gravures continentales représentant la folie du monde et des hommes dont on retrouve l'influence dans l'Angleterre élisabéthaine et jacobéenne où, selon Anthony Wells-Cole, « in place of classical elegance, English patrons craved the Mannerist excess of Antwerp ${ }^{7}$ ». La dialectique de l'excès propre à l'école du Nord semble en effet assez proche en esprit de celle du drame shakespearien.

\section{Le miroir de la folie}

En dehors de l'excentricité formelle du maniérisme nordique, le thème de l'excès dans l'art pictural du Nord est lié à la représentation de l'homme pécheur. L'énigmatique hybride de Jérôme Bosch s'inscrit dans cette tradition dont on retrouve les traces dans The Anatomy of Melancholy de Robert Burton. En effet, dans l'adresse de Démocrite au lecteur, l'auteur propose une somme de la folie humaine, dans laquelle il invite ses contemporains à se reconnaître ${ }^{8}$. Chacun a sa part de folie

1650 " in A Companion to English Renaissance Literature and Culture, éd. Michael Hattaway, Oxford, Blackwell, 2000, p. 442-63.

7 Ibid $\mathrm{n}^{\circ} 4$, p. 42 et p. 300 . Après le livre d'Anthony Wells-Cole, la publication en $2000 \mathrm{du}$ facsimilé du manuscrit The Great Book of Thomas Trevilian (1608) fournit des indications précieuses sur la diffusion des motifs continentaux utilisés pour les tissus peints. Les allusions sont fréquentes dans le corpus shakespearien, mais on en trouve également chez John Donne, Thomas Nashe, Ben Jonson, Middleton, Chapman, Marston et Robert Burton. Henry Peacham, quant à lui, conseille au parfait gentleman d'acquérir une connaissance approfondie de la gravure, et il recommande l'œuvre gravé d'Hendrick Goltzius à titre d'exemple des «principall Authors for your imitation », tout comme son « honest loving friend Crispin de Passe of Utrecht ». Il renseigne également sur les lieux où se procurer les gravures : "For a bold touch, variety of posture, curious and true shadow, imitate Goltzius, his prints are commonly to be had in Popes-head-alley » (passage tiré du Graphice, 1606, repris dans The Gentlemans Exercise, 1612 et 1634). J'adresse à Malcolm Jones, de l'Université de Sheffield, mes remerciements pour ces informations. Ce dernier met également au jour des emprunts que les Anglais auraient faits à certains scènes d'allégories obscures de Goltzius qui datent de sa période de collaboration avec Dirck Volckertszoon Coornhert. Voir à ce sujet, Malcolm Jones, English Broadsides-I, Print Quarterly, XVIII, 2001, 2, p. 149-63)

8 Voir aussi «The Argument of the Frontispiece», vii. «But see the madman rage downright / With furious looks, ghastly sight. / Naked in chains bound doth he lie / And roars a main, he knows not why. / Observe him ; for as in a glass, / Thine angry portraiture it was. His picture keep still in thy presence ; / Twixt him and thee there's no difference ». La description des différents symptômes de la mélancolie se clôt sur la description de la condition humaine: "Which (symptoms) I have been the most curious to express and report, not to upbraid any miserable man, or by way of derision (I rather pity them), but the better to discerne, to apply remedies unto them ; and show [...] how much we ought to fear our fickle estates, remember our miseries and vanities, examine and humiliate ourselves, seek God, and call to him for mercy : that needs not look for any rods to scourge ourselves, since we carry them in our own bowels... ", Op.cit, n ${ }^{\circ}$ 6, part I, p. 408. 
et nul n'y échappe, pas plus qu'à ses excès. Si l'on considère l'ensemble des éléments présents sur la gravure réalisée d'après le dessin de Bosch, on voit qu'il s'agit là d'une énumération de différentes formes d'excès (fig.1).

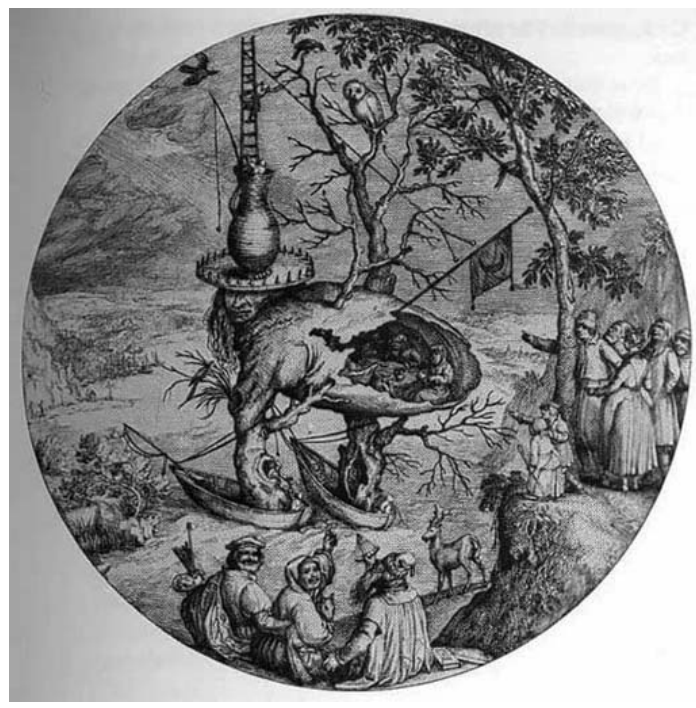

Fig.1. L'hom me-arbre, gravure anonyme, milieu Xvie siècle, Noordbrabants Museum, Hertogenbosch

La chouette perchée sur l'arbre desséché symbolise la folie et l'erreur' ${ }^{\text {. }}$ L'homme-arbre a un corps en forme de coquille vide, à l'intérieur de laquelle on aperçoit (comme dans la partie infernale du triptyque du Jardin des délices du Musée du Prado à Madrid) une scène de taverne, ou merry company, dont la joyeuse débauche vient dénoncer la

${ }^{9}$ L'oiseau surprend également par son regard observateur et c'est, là encore, l'image de la folie qui se contemple. Selon certains historiens d'art, l'oiseau de nuit représentait également la conscience de la folie et de l'introspection. Dirk Bax, Hieronymus Bosch. His Picture Writing Deciphered, Rotterdam, 1979, p. 191 et 264. Cette symbolique coïncide avec celle du fou dans l'art graphique. Les deux troncs représentant les jambes de l'homme arbre reposent à l'intérieur de deux petites barques, qui reprennent le thème de la barque de la Nef des fols, barque instable d'une vie au cours incertain à laquelle Timon fait aussi référence " That nature's fragile vessel doth sustain / In life's uncertain voyage » Timon of Athens (v.ii.86-87). 
gloutonnerie, les beuveries et la luxure ${ }^{10}$. Ces mêmes scènes de genre propres à l'art du Nord émaillent les récits shakespeariens. Hamlet se désole de l'ivrognerie et des autres excès qui sévissent à la cour du Danemark, tandis que l'intendant de Timon s'indigne des « riotous feeders » et «drunken spilth of wine » et se demande " How many prodigal bits have slaves and peasants / This night englutted» (II.ii.164-5). Il fait ici directement référence au péché de gourmandise, péché mortel connu au Moyen Âge sous le nom de Gula ${ }^{11}$. Chez Bosch, le corps ou le torse de l'œuf-taverne rappelle d'autres créatures drolatiques qui semblent n'être qu'un ventre et où les bas instincts ne sont pas soumis à la raison ${ }^{12}$.

Pour ce qui est de la raison revenons-en à la gravure et au motif de l'échelle. Ce motif à forte portée symbolique a toujours été populaire, et dans la culture chrétienne il prend une place très importante. Christian Heck distingue deux tendances dans l'utilisation de cette image. La première, l'échelle cosmologique, sert à penser le devenir de l'âme, son ascension hors du corps jusqu'au lieu du jugement par Dieu ${ }^{13}$. La deuxième, l'échelle spirituelle, figure la

${ }^{10}$ John Donne fait référence à ce type d'images négatives : «To teach by painting drunkards, doth not last / Now Aretines pictures have made few chast; / No more do Princes courts, though there be few, / Better pictures of vice, teach me vertue; » dans The Works of John Donne, The Wordsworth Poetry Library, Hertfordshire, Wordsworth Editions, 1994, Satyre IIII, (69-72).

${ }^{11}$ Le motif de la coquille vide, assez commun dans les tableaux de Vanités du XVII ${ }^{\mathrm{e}}$ siècle, a été utilisé par Bruegel dans une gravure, Fool on an egg, qui fait partie d'une série de proverbes proposés en tondo. L'ivrognerie y est dénoncée par son image car elle mène à la ruine matérielle et spirituelle, exprimé à travers le motif du fou et de la coquille vide. L'œuf était un symbole de gloutonnerie, lié aux festivités de Mardi gras, ainsi que de folie et de luxure. Op. cit., p. 302. Hamlet compare le territoire convoité par les Polonais et Fortinbras à une coquille d'œuf. L'image est alors symbole de ce qui est dérisoire et vain. Voir aussi François Laroque, "Pratique et esthétique de la subversion dans Hamlet », in Actes du colloque Hamlet, éd. G. Mathis, Aix-en-Provence, 1997.

12 On les retrouve chez Bruegel, Bosch et Alart du Hamel. Dans une gravure de ce dernier, «St Christophe », ces hommes hybrides peuplent la rivière et les berges aux côtés de personnages portant un habit de fou. Sur la berge vers laquelle saint Christophe se dirige, on apercoit un ermite qui porte une lampe en plein jour. Selon le catalogue de l'exposition, In Buscoducis, il s'agit d'un ermite qui montre le chemin à suivre, ou encore du motif de Diogène qui a bien du mal à trouver un " homme » au milieu de tous ces monstres et ces fous. Les monstres inspirés des œuvres de Bosch ressemblent aux êtres hybrides des Songes drolatiques de Pantagruel, (Voir l'édition de Michel Jeanneret, La Chaud-de-Fond, 1989). Cette gravure, datant de la fin du XVe siècle, imprimée sur du papier datant du XVII ${ }^{\mathrm{e}}$ siècle, reprend ce thème de la folie du monde illustrée par les motifs de la barque et des fous, les êtres hybrides, les échelles qui se retrouvent en partie dans l'étrange dessin de Bosch.

13 Elle peut alors devenir l'instrument du jugement de l'âme, dont l'ascension peut être suivi d'une chute brutale si elle n'est pas digne du salut. 
progression de l'âme avant la mort vers la conversion intérieure ${ }^{14}$. La symbolique de l'échelle de la progression spirituelle, de la vertu ou du salut, également présente dans la célèbre Melancolia d'Albrecht Dürer, a été subvertie dans la gravure ${ }^{15}$. En effet, chez Bosch, l'avant-dernière marche est reliée par une ligne diagonale à un mât surmonté d'un drapeau orné d'un croissant de lune. Ce mât est planté dans l'œuftaverne. Le croissant de lune rappelle l'inconstance humaine dans l'œuvre de Bosch où ce symbole est souvent associé à des scènes d'excès. Assurément alors, les orgueilleux et les savants ambitieux s'élevant de la cavité creuse de la cruche se retrouvent à bord d'un même bateau en route vers la perdition que ceux qui s'adonnent aux plaisirs de la chair ${ }^{16}$. Le motif de la canne à pêche est ainsi détourné de son sens initial, à savoir amener les âmes vers la vraie religion. Elle complète l'analyse de l'image et devient un symbole des tentations terrestres dont les démons accablent les hommes ${ }^{17}$. Shakespeare emploie cette image dans Measure for Measure : « O cunning enemy, that, to catch a saint, / With saints dost bait thy hook! Most dangerous / Is that temptation that doth goad us on / To sin in loving virtue » (II.ii.185-88). L'homme hybride de Bosch figure en quelque sorte le

\footnotetext{
14 À propos du motif de l'échelle voir Christian Heck, L'Échelle céleste dans l'art du Moyen Âge. Une image de la quête du ciel, coll. "Idées et recherches », Paris, Flammarion, 1997 Émile Mâle, dans L'art religieux au XIII ${ }^{e}$ siècle en France, 2 vol., Paris, Armand Colin, 1951, reproduit en illustration une échelle de la Vertu, tirée de l'Hortus deliciarum. L'échelle traverse l'image en diagonale, de gauche à droite. En bas figure un monstre infernal, tandis qu'en haut on devine une couronne céleste. Les personnages placés sur l'échelle peuvent représenter les différents âges de l'homme. La plupart d'entre eux font une chute vertigineuse, se laissant tenter par les biens temporels figurés en contrebas et poussés par les diables avec leurs arcs représentés à gauche de l'échelle.

15 Jérôme Bosch place son échelle au sein d'une cruche juchée sur la tête du personnage hybride. La cruche ainsi remplie s'avère être une disposition de mauvais augure qui dénote dans bien des tableaux et gravures la luxure et le lieu de mauvaise vie, comme la cruche sur la tête d'un personnage dénote la folie. Cette folie fait apparaître l'échelle comme celle de l'ambition et de l'orgueil de ceux qui tentent de s'élever toujours plus haut dans la hiérarchie terrestre. Elle est alors symbole de tromperie et d'illusion. Voir Bax, Op. cit. $\mathrm{n}^{\circ} 9$, p. $128,130,146$.

16 Comme chez Rabelais, pour qui, comme on sait, "science sans conscience n'est que ruine de l'âme ", Livre II, Pantagruel, chapitre 8, Rabelais Euvres complètes, éd. Guy Demerson, Paris, Seuil, 1973, p. 248.

17 Nous le rencontrons assez fréquemment chez Bosch et chez d'autres graveurs, mais la signification la plus explicite se trouve dans une gravure de Coornhert. Nous y voyons l'apôtre Pierre au-dessus d'un globe dans lequel des hommes nagent. Il tente de pêcher des âmes à l'aide d'une canne pourvue d'un hameçon en forme de crucifix. De l'autre côté, un ange déchu assis sur la bouche de l'enfer fait de même et il a visiblement plus de succès puisqu'il utilise comme hameçon les symboles de l'amour charnel, de la gloutonnerie, de la beuverie, et de la richesse. L'homme qui saisit la bourse d'argent est coiffé d'un globe terrestre.
} 
portrait du « common sinner » que Hamlet adjure Ophélie d'éviter de mettre au monde ${ }^{18}$.

L'écart entre le dessin initial de l'homme-arbre, un temps attribué à Bruegel, et la gravure du milieu du $\mathrm{XvI}^{\mathrm{e}}$ siècle montre un choix ultérieur de mise en scène de théâtre. Cette mise en scène permet une mise en abyme à valeur édifiante. En effet, un groupe de spectateurs représentant plusieurs groupes sociaux ainsi que la figure du peintre lui-même ont été rajoutés sur la gravure. La présence de spectateurs est un motif récurrent dans le corpus d'images traitant des excès de l'homme. Un garçon montre l'étrange figure à une petite fille. Le motif de l'enfant spectateur de la bêtise des adultes, motif que l'on rencontre dans certaines scènes de genre, se retrouve dans la réplique que Warwick fait au roi Henri IV: "The Prince but studies his companions... » (2 Henry IV, Iv.iii.64-70), ce qui suggère que Hal se livre à une étude sur modèle vivant dont il pourra tirer profit par la suite $^{19}$. Dans l'image, le groupe d'hommes et de femmes spectateurs qui montrent également du doigt l'homme-arbre en riant remplacent le traditionnel bouffon sage et indiquent un spectacle satirique et édifiant proche en esprit des «thousand moral paintings » dont il est aussi question dans Timon of Athens ${ }^{20}$. La position excentrée, en retrait par rapport aux excès du monde de ces spectateurs, est traditionnellement celle du bouffon qui, tel Jaques ou Hamlet, revendique la liberté de fustiger le monde du bois vert de sa satire. Cependant, les spectateurs ne sont pas les seuls à nous regarder. L'homme-arbre porte lui aussi les yeux sur nous, et ce regard dirigé vers le spectateur produit un peu l'effet de quelqu'un qui se regarderait dans un miroir. Cet effet se trouve renforcé par le choix d'une gravure en médaillon ou tondo. Dans le jeu des regards entre l'homme hybride et le spectateur, l'action de se

18 «Why wouldst thou be a breeder of sinners » (III.i.123). Dans le tryptique du Jardin des Délices, l'homme arbre trouve sa place au sein du volet gauche, l'Enfer.

19 « The Prince but studies his companions / Like a strange tongue, wherein to gain the language, / 'Tis needful that the most immodest word / Be looked upon and learnt, which once attained, / Your Highness knows, comes to no further use / But to be known and hated. So like gross terms, / The Prince will in the perfectness of time, / Cast off his followers, and their memory / Shall as a pattern or a measure live / By which his grace must mete the lives of other, / Turning past evils to avantages. (IV.iii.64-70). Un autre exemple est la réplique ironique dans Thomas Middleton, A Trick to Catch the Old One: "She that knows sin knows best how to hate sin », (v.ii.132).

${ }^{20}$ Op. cit., $\mathrm{n}^{\circ} 3$, (I.i.91). 
mirer vaut pour l'un autant que pour l'autre : nous voici face au miroir de la folie qui nous renvoie à nos excès ${ }^{21}$.

Cette façon plutôt mélancolique d'appréhender le monde caractérise Hamlet et Timon qui se convainquent de la nature irrémédiablement déchue de l'homme. Les deux personnages semblent ne retenir de l'homme que scènes de débauche et de mensonge. Hamlet s'accuse des pires abominations, ne reconnaît plus de vertu à Ophélie et il se complait dans des piques grivoises plutôt grossières à son égard car la femme est à ses yeux l'image même du péché ( « frailty, thy name is woman ", I.ii.146). Timon, quant à lui, endosse l'habit du misanthrope au point que, dans son rejet de l'humanité tout entière, de lui-même y compris, il a du mal à reconnaître un homme honnête en la personne de son intendant. Il n'est pas anodin que cette personne, tout comme Horatio dans Hamlet, soit désignée comme étant dépourvue de cette richesse qui brouille les frontières entre l'être et le paraître et qui contient en son cœur le germe de sa propre déchéance. Les thèmes et motifs graphiques et littéraires sur l'excès vont en effet commenter une mutation du monde liée à l'essor du capitalisme qui entraîne une abondance culpabilisante décrite par Simon Schama ${ }^{22}$ comme l'embarras de richesses ou encore par Costard dans Love's Labour's Lost, comme « the sour cup of prosperity. » (I.i.287).

\section{Les cinq sens et les tentations d'un nouveau monde}

Le thème des cinq sens se retrouve, lui aussi, dans Timon of Athens, où Cupidon fait de ces figures une allégorie de l'opulence et de la richesse :

The five best senses

Acknowledge thee their patron, and come freely

To gratulate thy plenteous bosom. Th'ear,

\footnotetext{
${ }^{21}$ Selon Érasme, la folie est en effet le moins menteur des miroirs de l'âme. Dans L'Éloge de la folie, (v) et (xxxvi), les fous sont les seuls sincères et véridiques. Érasme. Éloge de la folie, Adages, Colloques, Réflexions sur l'art, l'éducation, la religion, la guerre, la philosophie, Correspondance, éd. Claude Blum, André Godin, Jean-Claude Margolin et Daniel Ménager, Paris, Robert Laffont (Bouquins), 1992. Le miroir satirique d'Horace et celui de Sénèque, celui-là même qui reflète sa propre folie, et auquel Robert Burton ne manque pas de faire référence: "When I would solace myself with a fool, I reflect upon myself, and there I have him » ( « Democritus to the Reader », op. cit, $n^{\circ}$ 6, p. 77).

${ }^{22}$ Simon Schama, L'embarras de richesses, trad. Pierre-Emmanuel Dauzat, Paris Gallimard, 1991. Titre original, The Embarrassment of Riches, New York, Alfred Knopf, 1987.
} 
Taste, touch, smell, all, pleased from thy table rise.

They only now come to feast thine eyes.

(I.ii.120-24)

Il y a là un raffinement qui caractérise également les représentations des cinq sens personnifiés par des allégories féminines dans l'univers graphique de la fin du XVI ${ }^{\mathrm{e}}$ et au début du XVII ${ }^{\mathrm{e}}$ siècle. L'accroissement de l'offre de produits de consommation provenant de l'artisanat et de l'agriculture, que l'on voit étalées dans une profusion gaspilleuse sur les tableaux représentant des scènes de cuisine ou des scènes de marché, éveillant, certes dans la sphère de la fiction, les envies au moyen d'illusions, produisirent une profonde restructuration de la sensualité. Les séries de gravures et de tableaux réalisés autour du thème des cinq sens montrent que l'offre de marchandises de luxe déclenche chez l'observateur un besoin forcené de céder à ses envies et à un accroissement permanent de ses besoins ${ }^{23}$ ( « I dreamt of a silver basin and ewer tonight » Timon, II.i.5). Elles illustrent une inquiétude face aux excès possibles et se trouvent ainsi reliées au thème du miroir de la folie. Une estampe d'une scène de genre avec couples d'amoureux du début du $\mathrm{XVII}^{\mathrm{e}}$ siècle sera ainsi adaptée pour représenter les cinq sens (fig. 2 et 3 ).

Fig.2. Intérieur avec couples d'amoureux, Johan Liss, début XVII' siècle, Cabinet d'estampes, Rijksmuseum, Amsterdam

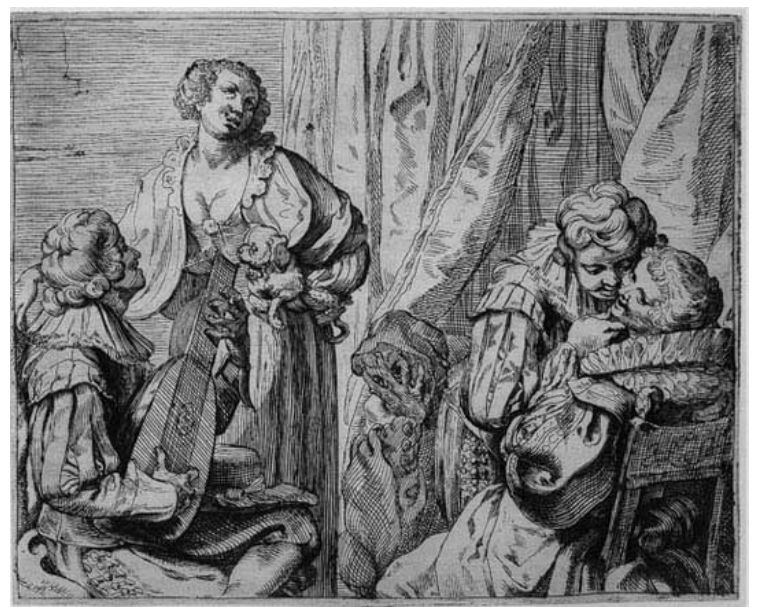


Fig.3. Les cinq sens, Cornélis Galle d'après Johan Liss, début XVII siècle Cabinet d'estam pes, Rijksmuseum, Amsterdam

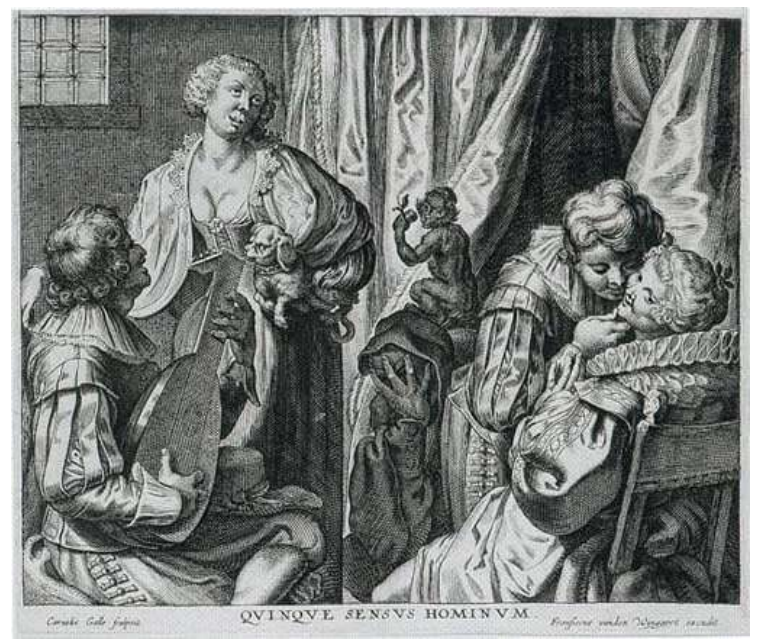

Le motif du bouffon qui regarde à travers ses doigts remonte à la tradition inaugurée par la Nef des fous et signe les satires de l'indulgence coupable envers le monde et sa séduction fallacieuse ${ }^{24}$ (« Hey-day, what a sweep of vanity comes this way », Timon, I.ii.128). Les tableaux peints par Jan Bruegel l'Ancien sur le thème des cinq sens illustrent le fait que l'unité de la personne se trouve divisée en parties sensorielles correspondant à une différenciation croissante de la réalité définie en domaines fonctionnels par des biens de luxe. La série des cinq sens gravée d'après Hendrick Goltzius est proche de cette tradition (fig. 4 a-e). Dans certaines de ces représentations, la découverte et la compréhension du monde s'effacent au profit d'une réception sensuelle parfois mise en scène comme un moment de possession érotique par des personnages féminins, en partie dénudés et placés au sein d'un écrin de douce opulence.

23 Norbert Schneider, Les Natures mortes, Réalité et symbolique des choses, Cologne, Taschen, 1991, p. 65.

24 Ger Luijten, Mirror of Everyday Life,Genreprints in the Netherlands 1550-170o, Catalogue d'exposition, Rijksmuseum Amsterdam, Gand, Snoeck-Ducajoe \& Zoon, 1997, p. 154 . 
Fig.4a-e. Les cinq sens, Série de gravures de Jean Saenredam (1565$1607)$ d'après Hendrick Goltzius, F.W.H Hollstein :Dutch and Flem ish Etchings, Engravings and Woodcuts, ca. 1450-1700, vol. viii, Amsterdam 1949-87, Roosendaal, 1988 -94
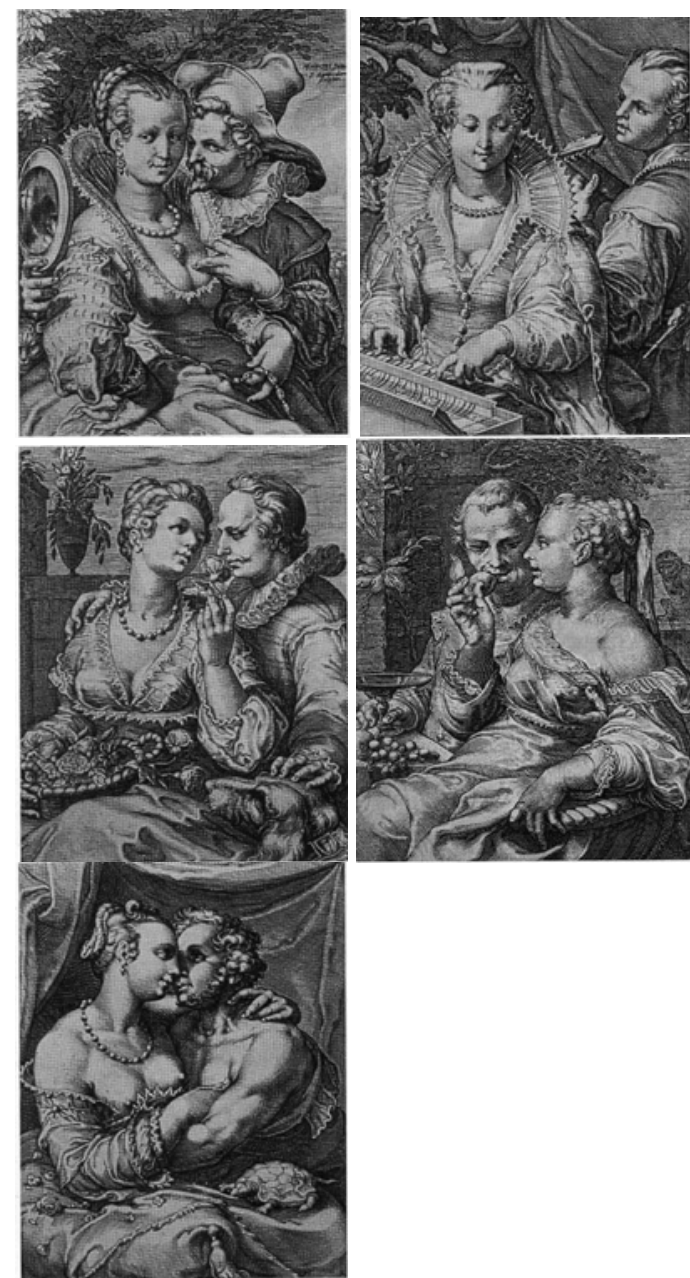
Chez Timon, les cinq sens s'inscrivent dans cette «softness of prosperity » dont le poète propose la satire (IV.ii.34) et qui, selon certains, émousserait les sens.

Certains critiques ont noté que Timon of Athens repose plus sur des schémas de pensée éthique que sur la caractérisation des personnages $^{25}$. La réplique du poète à propos de sa satire à la fin de la pièce donne un écho ironique aux «thousand moral pictures » du début. Les deux passages renvoient à un même arrière-plan de lieux communs imagés dont les exemples picturaux suivants donnent un aperçu (fig. 5 a et b).

Fig.5a et b. Dangers inhérents à la Richesse et Conséquences de la prospérité, Philips Galle d'après Maarten van Heemskerck, 1563, issue de la série Les malheurs de la prospérité, Cabinet d'estampes, Rijksmuseum, Amsterdam
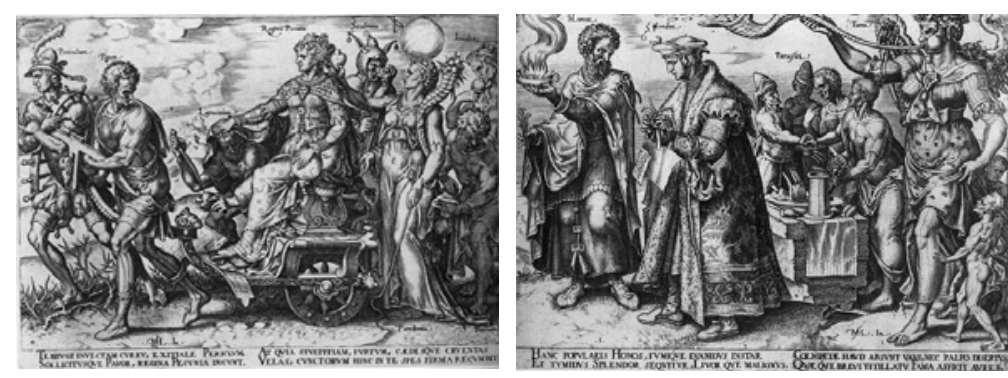

Dans Dangers inhérents à la Richesse et Conséquences de la prospérité toutes deux issues de la série Les malheurs de la prospérité (1563), Maarten van Heemskerk visualise certains propos récurrents au sein du texte shakespearien. Regina Pecunia, la reine de l'argent, est représentée assise sur son char de triomphe et, sous sa robe, elle cache Meurtrière Rapine (Latrocinium). Quant à sa main posée sur un globe terrestre, elle signifie le pouvoir qu'elle exerce sur le monde. La reine est accompagnée de Pandemia (le monde entier) placée sous son emprise et elle porte sur la tête le symbole du monde à l'envers rappelant l'or qui dans Timon rend blanc le noir, beau le laid et juste l'injuste. Son char est suivi par la Sottise, le Vol et l'Envie. Sur la

25 Op. cit, $\mathrm{n}^{\circ} 3$, p. 883. 
deuxième gravure, on reconnaît les personnifications qui accompagnent la prospérité. On voit aussi l'Honneur et Splendeur, tous deux éphémères, comme la Renommée, symbole du paraître, armée d'une trompette qui semble semer des langues. À l'arrière-plan, les Parasites se disputent les plats.

Fig.6. Om nium rerum vicissitudo est, Zacharias Dolendo d'après Jacques de Gheyn II, 1596, cabinet d'estampes de l'Université de Leiden

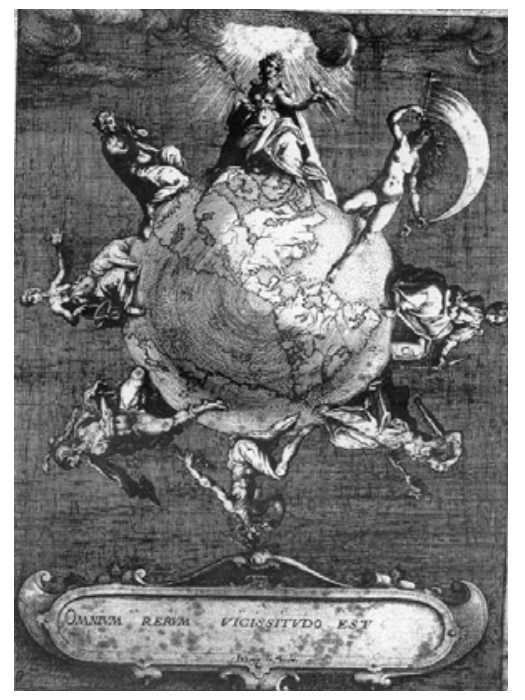

Cette série d'images, proche du Cycle des vicissitudes humaines, appelé aussi le Cycle de la guerre et de la paix, sera régulièrement utilisée par les artistes graveurs, les peintres et les poètes. Le motif graphique propose une somme des agissements humains traversant sept phases différentes (fig.6) ${ }^{26}$. De la Prospérité

${ }^{26}$ La première gravure d'une série réalisée par Jacques de Geyhn sur ce thème révèle la relation que le concept du cycle entretient avec la roue de la Fortune. Cependant, la rotation du monde est ici le fait des excès de l'homme. De là peut-être une partie de l'ironie aux dépens de Rosencrantz et Guildenstern lorsqu'ils se félicitent d'être " As the indifferent children of the earth. / Happy in that we are not over-happy » (II.2.229-30), non au faite de la Fortune mais en son « juste » milieu : " In the secret parts of Fortune ». Ils sont porteurs de la nouvelle « the world's grown honest », proclamation dont le burlesque n'échappe ni à Hamlet ni au public. 
procède l'Orgueil, de l'Orgueil procède l'Envie, de l'Envie procède la Guerre, de la Guerre procède la Pauvreté, de la Pauvreté procède l'Humilité, de l'Humilité procède la Paix et de la Paix procède la Prospérité. L’idée sert de leitmotiv dans Coriolanus, ce qui n'empêche pas le dramaturge de tourner en dérision l'adage connu par un raccourci facétieux, « The wars for my money »(Coriolanus, IV.v.38) ${ }^{27}$. Shakespeare avait déjà fait référence au cycle à la fin de Timon of Athens :

AlCIBIADES. [...] Bring me into your city, And I will use the olive with my sword Make war breed peace, make peace stint war, make each Prescribe to other as each other's leech.

Le lieu commun reprend l'idée de l'excès qui contient en lui-même son propre déclin, sa propre "guérison » par le mal ou les vices même qu'elle entraîne en son sillage, en l'occurrence la guerre. Les différentes phases dans Timon, la prospérité, la ruine et la pauvreté et à nouveaux la richesse, même si celle-ci est refusée par Timon, qui ramène les parasites et les arts libéraux compagnons habituels de la Prospérité, se conforment en partie au cycle ${ }^{28}$. La présence de l'homme de guerre Alcibiade peut alors s'expliquer par la nécessité de l'équilibre instable ( "Sound this coward and lascivious town / Our terrible approach » (v.iv.1-2) qui corresponde au schéma de pensée du cycle, tout comme les mots de la fin inscrivent la pièce définitivement dans les vicissitudes d'un monde dont la première scène emblématique propose la peinture. « How goes the world ? [...] It wears, sir, as it grows. » (I.i.3-4).

\section{La dialectique de l'excès prise en défaut}

Malgré les efforts des peintres et des poètes, Timon est un personnage qui reste aveugle au monde. En cela, il ressemble un peu à Falstaff qui n'a jamais fait mentir de proverbe. En effet, dans la série de proverbes gravés d'après de Pieter Bruegel en 1563 intégrée aux Emblemata

\footnotetext{
${ }^{27}$ Voir à ce propos, Nuyts-Giornal, " "The wars for my money” : La politique morale du " painted cloth » dans Coriolan de Shakespeare » in Richard Hillman éd., Coriolan de William Shakespeare, Actes du Colloque de l'Université François Rabelais, 3-4 novembre 2006, Tours, Presses Universitaires François Rabelais, 2007, p. 81-111.

${ }^{28}$ Nous retrouvons également le thème de l'usure qui permet de s'enrichir en temps de paix, remis en cause dans le cycle des vicissitudes : " I have kept back their foes / While they have told their money and let out / Their coin upon large interest » (III.vi.104-106).
} 
saecularia de Théodore de Bry en 1611, deux personnages stéréotypés rappellent les tribulations de Timon (fig. 7 a et b).

Fig.7. Proverbes, gravures d'après Pieter Bruegel L'Ancien (1568-

1611) dans Théâtre d'Amour, réimpression des Emblem ata amatoria de 1620 , éd. Carsten-Peter Warncke, Paris, Taschen, 2004 , folio 83 et 88
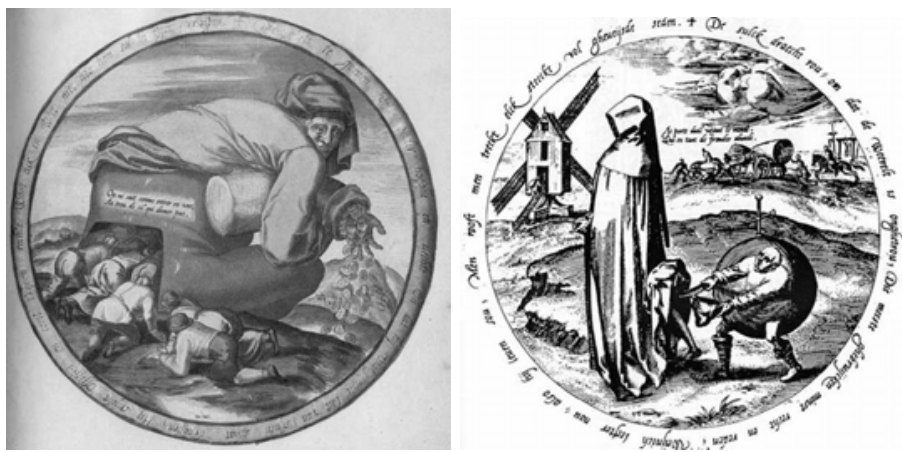

Sur l'une des gravures en tondo, des flatteurs rampent dans le cul d'un riche dans l'espoir de recevoir une part des pièces d'or que le géant répand de la main. Sur l'autre, le Misanthrope tourne le dos au monde perfide qui au passage lui vole sa bourse. Le texte shakespearien emploie des images analogues. Ainsi à propos de Timon, « He pours it out. Plutus the god of gold / Is but his steward », (I.i.279). Apemantus remarque « O you gods, what a number of men eats Timon, and he sees 'em not! ». Son intendant déclare regretter que «'Tis a pity Bounty had not eyes behind » (I.ii.161). Et, dans la deuxième partie de la pièce, Timon, s'en va seul comme le mépris emportant avec lui les faux serments de ses anciens amis «like empty purses pick'd» (IV.ii.12). Dans les deux cas, le personnage tourne le dos au monde et ne peut donc apprendre de lui, ignorant les préceptes humanistes et rendant vains les efforts du poète et du peintre.

Drapé dans une même mélancolie de misanthrope, Hamlet est cependant présenté par le dramaturge comme un homme qui s'observe et observe les autres selon les préceptes humanistes qui enseignent la connaissance de soi et du monde. D'une certaine manière, comme le 
couple qui désigne de la main une scène de théâtre sur la gravure d'après David Vinckboons, il sait les leçons de mesure, même lorsqu'elles sont amères, qu'il convient de tirer des excès et du grouillement humain observé dans les scènes de kermesses (fig. 8).

Fig.8. Kermesse, Johannes Swaneburg d'après David Vinckboons, 1610 , Cabinet d'estampes, Rijksmuseum, Amsterdam

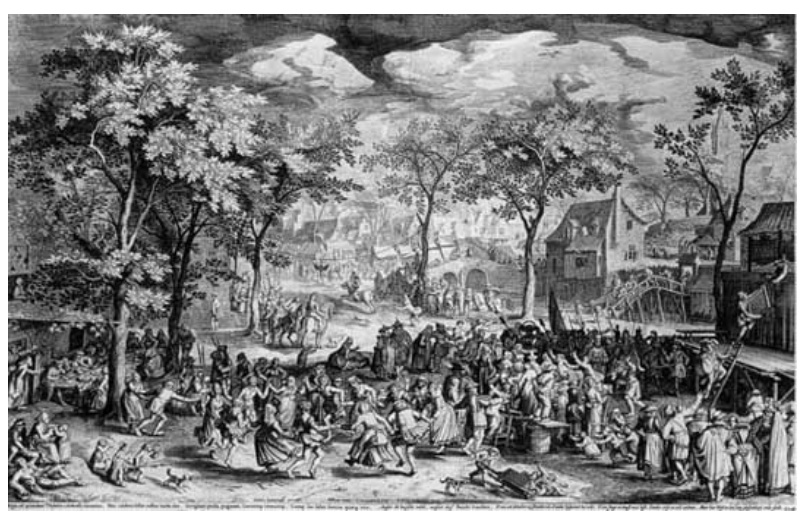

À cet égard, le prince mélancolique ressemble à Marc Antoine qui, au début d'Antoine et Cléopâtre, propose à la reine d'aller observer les gens dans les rues et qui saura par la suite nommer sa propre déchéance et sa fragilité ${ }^{29}$. Le dramaturge montre aussi un personnage qui, à l'instar d'Hamlet, se méfie des cinq sens et de ses propres turpitudes comme cela est enseigné dans la gravure morale. La série de gravures sur cuivre d'après Hendrick Goltzius, déjà mentionnée, prétend aussi exhorter le spectateur à prendre conscience des dangers inhérents aux cinq sens qui devraient permettre à l'homme de distinguer le bien du mal mais qui sont également la voie par laquelle le goût du péché pénètre l'âme ${ }^{30}$. Les images montrent des couples en train de s'ébattre dans un décor raffiné dont le luxe chatoyant renforce le caractère assez fortement érotique (fig. 4). À en croire les

${ }^{29}$ Nuyts-Giornal, « Genre-print and Early Modern English Literature », Cahiers Élisabéthains, $\mathrm{n}^{\circ}$ 70, Montpellier, 2006, p. 21-31. Dans La Tempérance, gravure d'après Bruegel issue de la série des sept vertus, on aperçoit également une scène de théâtre avec un bouffon qui contemple sa marotte.

30 Op.cit. $\mathrm{n}^{\circ}$ 24, p. 241. 
affirmations des titres des gravures, cette douce opulence favorise le vice et, au lieu du plaisir, elle ne suscite que le dégoût. Ici encore, l'unité sensorielle de la personne est divisée en parties différenciées par les vices auquel l'homme se complait à succomber ${ }^{31}$. Chez le philosophe et théologien Nicolas de Cuse (1401-1464), l'être de sens parfait reposait sur l'unité sensorielle de la personne qui permet de découvrir et d'appréhender le monde ${ }^{32}$. Hamlet fait allusion à cet être de sens parfait: "In apprehension how like a god, the paragon of animals » (II.ii.8). Par la suite, son incompréhension face aux errements sensuels de sa mère s'exprime par une réflexion sur les cinq sens pris en défaut de production de sens. L'image utilisée est alors celle de l'éclatement de l'unité sensorielle propre aux gravures qui représentent les cinq sens en figures indépendantes :

Sense sure you have,

Else could you not have motion ; but sure that sense

Is apoplexed, for madness would not err,

Nor sense to ecstasy was never so thralled

But it reserved some quantity of choice [...]

Eyes without feeling, feeling without sight,

Ears without hands or eyes, smelling sans all,

Or but a sole part of one true sense

Could not so mope.

(Q2, III.iv.70)

Le terme «apoplexed» correspond au résultat d'un excès. Dans Coriolan, il est lié à l'abondance et la prospérité du temps de Paix, cette dernière est décrite comme « a very apoplexy, lethargy ; mulled, deaf, sleeping, insensible... » (IV.v.228). La hantise qu'éprouve Hamlet face au vice consubstantiel à l'homme se traduit de façon récurrente par ce réseau d'images. Ainsi, " the fatness of these pursy times » (III.iv.144) qui obligent la vertu à s'incliner devant le vice traduit la même idée et rejoint ses réflexions à propos de la guerre qui serait déclenchée pour

${ }^{31}$ Dans «La Vue », on prévient contre le débridement du petit œil « ocelli », L’Ouie, symbolisé par la viole et le luth dont joue le jeune couple, est associé au sons de sirènes flatteuses, L'Odorat est illustré par un jardin fleuri et une jeune femme qui fait sentir une rose à son compagnon. Le Goût est symbolisé par des fruits et du vin pour conjurer selon le titre de la gravure l'intempérance qui, jusqu'au Moyen Âge, faisait encore figure de péché mortel. Le Toucher est, lui, représenté par un couple en train de s'embrasser. La série s'apparente ainsi au thème de "Sine Cerere et Baccho friget Venus », sujet qui était parmi les favoris d'Hendrick Goltzius.

${ }^{2}$ Norbert Schneider, Les Natures mortes. Realité et symbolique des choses, Taschen, Cologne, 1991, p. 65. Le philosophe compare l'être de sens parfait à un cosmographe possédant une ville à cinq portes par lesquelles arrivent des messagers arrivant du monde entier entrent et relate tout le développement du monde. 
un fétu de paille ou une coquille d'œuf : «This is th'imposthume of much wealth and peace / That inward breaks and shows no cause without / Why the man dies. » (IV.iv.25). Le prince mélancolique est ici au faîte du répertoire du "painted cloth » et de son discours sur les excès et les fourberies du monde ; malgré tout, le dramaturge montrera ironiquement comment Hamlet se laisse lui aussi prendre au piège du sens forcément limité que l'homme a des choses et de lui-même.

Une série d'images gravées issues d'un album d'Emblemata montre les cinq sens accompagnés d'un quatrain rédigé chacun en une langue différente. L'Odorat représenté par une rose (et non une tulipe) est assorti à un quatrain en hollandais, L'Ouïe montre un luth et le quatrain est en français. La Vue est accompagnée d'un miroir et de vers en latin. Le Goût porte une coupe et propose un quatrain en allemand (les excès dans les plaisirs de la bonne chère et de la boisson faisaient partie des reproches souvent faits aux Allemands). Le toucher s'affiche avec un perroquet et un quatrain en anglais. Tous nous exhortent à nous contenter de ce que nous sommes, tout comme des moyens qui sont les nôtres ${ }^{33}$. Sous toutes les latitudes, les cinq sens restent les cinq sens qui nous renvoient à notre subjectivité. Ainsi, Hamlet, en endossant le rôle du fou qui au travers de l'expérience théâtrale tend au monde un portrait cruel mais salutaire, accepte le sacerdoce humaniste qui consiste à vouloir rendre le monde meilleur mais il n'échappera pas pour autant à sa condition d'homme. Suivant le principe des miroirs de la folie, Shakespeare amène le Prince à faire siens les faits répréhensibles qu'il entend punir chez Claudius comme chez Gertrude. La prudence dont il fait preuve face à l'apparition du spectre puis lors de ses réflexions sur la meilleure action à mener ne lui épargneront pas des erreurs d'approximation grossières. Ainsi, il se trompe en croyant voir en Claudius agenouillé un pécheur repenti, il tuera Polonius à la suite d'une erreur d'appréciation et bafouera la vertu d'Ophélie, dont il a scruté le visage pour n’y découvrir que sa propre noirceur.

Dans la pièce dans la pièce, le dramaturge ébauche une réflexion sur son art et sur les clichés qui le désignent comme un genre moral. Hamlet relève toutes les qualités édifiantes et cathartiques habituellement prêtées à l'art théâtral : «Make mad the guilty and

33 Cinq sens, folios 106-111, série édité chez Assverus van Londerseel à Rotterdam ou Amsterdam, vers 1610 dans Théâtre d'Amour, réimpression intégrale des Emblemata amatoria de 1620, Essai et textes de Carsten-Peter Warncke, Taschen, Londres, 2004. 
appal the free » (II.ii.566) ou encore « The play's the thing / Wherein I'll catch the conscience of the King» (II.ii.607) en imitant son " humanité ». De façon docte, le Prince nous fait la leçon sur «the purpose of playing, whose end, both at the first and now, was and is to hold as 'twere a mirror up to nature, to show virtue her own feature, scorn her own image, and the very age and body of the time his form and pressure » (III.ii.20-25). En fait des traits déformés de la vertu, ce que reflète avant tout le miroir théâtral entre les mains du Prince, c'est la figure de Hamlet. Le protagoniste est victime de sa perspective subjective qui fait voir la vertu sous d'horribles aspects. Dans la littérature et l'art pictural, et par opposition à Dame Monde et à ses multiples attraits, la vertu est en effet souvent dépeinte comme austère, voire difforme, et généralement peu attrayante aux yeux du commun des mortels. Mais il s'agit là de la vision peu vertueuse que Hamlet était censé combattre ${ }^{34}$. L'homme, lucide au premier abord, s'est laissé entraîner malgré lui dans les excès de sa disposition mélancolique et ne sait plus vraiment distinguer le beau du laid.

Ainsi, au sein des miroirs satiriques déformants du bouffon ou du fou dans l'art graphique du Nord et dans le texte shakespearien, l'interrogation de la vérité demeure au cœur de la problématique d'une réflexion morale de type humaniste. Celle-ci semble ne dévoiler son véritable visage qu'au fou qui, comme Ophélie, en paye le prix, le prix de la raison. Restent en écho les chansons, les mots à rebours, les fatrasies et les coq-à-l'âne que le bouffon a empruntés, ainsi que sa position excentrée qui lui permet de railler les excès du Monde. Au terme de la pièce dans la pièce, Shakespeare conseille à son protagoniste de laisser courir le monde car, comme le dit Hamlet, "Would not this, sir, and a forest of feathers, if the rest of my fortunes turn Turk with me, with two Provençal roses on my rased shoes, get me a fellowship in a cry of players, sir ? " (Hamlet, II..ii.262-66). Sceptique quant aux fins édifiantes de l'art théâtral, Shakespeare, à l'instar de Sartre dans Les Mots, semble convenir du fait que « la culture ne sauve rien ni personne, elle ne justifie pas. Mais c'est un produit de l'homme :

34 Dans All's Well that Ends Well, «scornful perspective », «squint-eyed age ", " squint eyed soul » sont des termes utilisés pour dénoncer un regard de biais qui empêche d'aimer la vertu. On le retrouve chez Shakespeare, Thomas Dekker, et John Donne. Ces métaphores rappellent un peu le procédé de l'anamorphose dans Les Ambassadeurs de Hans Holbein. 
il s'y projette, s'y reconnaît; seul ce miroir critique lui offre son image ${ }^{35}$. $»$ Et, de fait le dramaturge semble bien adopter l'attitude équivoque présente dans certains autoportraits d'artistes du début de l'époque moderne ${ }^{36}$. La plume ou, à l'occasion, un verre à la main, ceux-ci s'affirment à la fois pleinement conscients de leur position dans le monde et du caractère universel de la portée de leur message moral et satirique.

Josée NUYTS-GIORNAL

35 Jean Paul Sartre, Les mots, Folio Gallimard, Paris, 1964, p. 205.

${ }^{36}$ Op. cit., $\mathrm{n}^{\circ} 29$, p. 28. 